\title{
Effects of surfactants on size and structure of amylose nanoparticles prepared by precipitation
}

\author{
YAN DONG, YANJIAO CHANG, QIAN WANG, JIN TONG and JIANG ZHOU* \\ Key Laboratory of Bionic Engineering (Ministry of Education), College of Biological and Agricultural Engineering, \\ Jilin University, 5988 Renmin Street, Changchun 130022, China
}

MS received 11 July 2015; accepted 31 August 2015

\begin{abstract}
The present work investigated the influence of surfactants on size and structure of amylose nanoparticles (ANPs) prepared through precipitation. ANPs were fabricated using absolute ethanol containing surfactants (Tween80, Span80 and mixtures of Tween80 and Span80 with ratios of 25/75, 50/50 and 75/25, respectively) as non-solvents. The obtained ANPs were characterized using dynamic light scattering (DLS), scanning electron microscopy and X-ray diffraction. The results showed that surfactant type, concentration and hydrophiliclipophilic balance (HLB) value had great impact on size of precipitated ANPs. The smallest ANPs with mean size of $155 \mathrm{~nm}$ determined by DLS were obtained by using $0.5 \%$ (in proportion of the amylose solution volume) of Tween80/Span80 mixture (HLB = 12.33). The precipitated ANPs possessed the V-type crystalline structure no matter whether using the surfactants or not.
\end{abstract}

Keywords. Amylose nanoparticles; surfactant; precipitation.

\section{Introduction}

Starch nanoparticles (SNPs) have practical or potential applications in many aspects, such as reinforcements for nanocomposites, emulsion stabilizer, adsorbents for wastewater treatment, etc [1-3]. Size and structure of SNPs are very important for their applications. Several processes have been tried out to produce SNPs, such as acid hydrolysis [4,5], precipitation [6-8], complex formation [9], high power ultrasonication [10], alkali-freezing treatment [11], enzymolysis and recrystallization [12]. Precipitation is a simple, fast and reproducible method to fabricate SNPs. This process involves a successive addition of a dilute starch solution to a nonsolvent or inversely. Nanoparticle formation via precipitation is assumed to be due to nucleation of small aggregates of macromolecules followed by aggregation of these nuclei [13].

Starch is a mixture of two polymers: linear amylose and highly branched amylopectin, except waxy starch which only contains amylopectin. Although fabrication of nanoparticles using a commercial native starch would be of relevance because of the lower cost as compared with prepared amylose, the molecular structure difference between amylose and normal starch could render difference on size, morphology and structure of the nanoparticles obtained by precipitation. On the other hand, since amylose is relatively simple and linear molecule, it tends to crystallize from solution by retrogradation. Understanding of the precipitation of amylose polymers from solution could provide insights for designing size and structure controlled nanoparticles made from amylose or

*Author for correspondence (jiang.zhou@jlu.edu.cn) native starch. Our previous work showed that the size of precipitated amylose nanoparticles (ANPs) was dependent on concentration of amylose solution, amount of used ethanol and mode of agitation during precipitation [14]. In this paper, operating parameters were maintained constant and ANPs were prepared through precipitation by using surfactants. The aim of this study is to investigate effects of surfactant type, concentration and hydrophilic-lipophilic balance (HLB) on size and structure of the precipitated ANPs.

\section{Experimental}

\subsection{Materials}

Amylose with content of $99.5 \%$ was purchased from Shaanxi Tianwei Biological Production Co. Ltd. (Xi'an, China). Tween 80 and Span 80 were produced by Tianjin Huadong Regent Factory (Tianjin, China). All the materials were used as received.

\subsection{Preparation of ANPs}

Amylose solution of $10 \mathrm{mg} \mathrm{ml}^{-1}$ was prepared by dissolving amylose in $90 \% \mathrm{DMSO} / 10 \%$ water mixture (v/v) at $90^{\circ} \mathrm{C}$ with continuous stirring of $200 \mathrm{rpm}$. The reason to use $90 \%$ DMSO/10\% water mixture (v/v) as solvent is that small amounts of water (5-15\%) tend to enhance the solubilization of starch in dimethylsulphoxide (DMSO) and amylose molecular chains can be dispersed better $[15,16]$. Non-solvents, absolute ethanol containing surfactants (Tween80, Span80 and mixtures of Tween 80 and Span 80 with ratios of 25/75, 
$50 / 50$ and $75 / 25$, respectively), were prepared by mixing absolute ethanol (double volume of the amylose solution to be precipitated) with the surfactants accounting for $0.5,1$, 2 and $4 \%$ of the volume of the amylose solution to be precipitated, respectively. After the obtained amylose solution reached room temperature, the prepared non-solvents were dropwise added into the amylose solution which was continually agitated with ultrasound. The resulting mixture was then centrifuged at $6000 \mathrm{rpm}$ and the precipitated ANPs were obtained by removing the supernatant and rinsed 3 times by centrifugation with absolute ethanol.

\subsection{Particle size analysis}

The mean size and size distribution of the prepared ANPs were estimated by dynamic light scattering (DLS) using a Malvern Zetasizer Nano-ZS90 (Malvern Instruments Ltd., UK). The measurements were performed with samples prepared by dispersing the obtained ANPs in deionized water at a weight ratio of about $1 / 500$. The mean size and polydispersity index (PDI, used to define the particle size distribution) were reported as the average of six measurements.

\subsection{Morphological studies}

Morphologies of the ANPs were observed using a Zeiss EVO 18 SEM (Zeiss, Germany). For the samples from precipitation, the powder was mounted on specimen stubs with carbon black tape and then sputter-coated with gold. For the samples re-dispersed in water, a droplet of the diluted suspension was placed on a silica surface, dried at room temperature and then sputter coated with gold.

\section{$2.5 X$-ray diffraction (XRD) analysis}

Crystalline structures of the obtained ANPs were characterized using a Rigaku D/max-2500 X-ray diffractometer (Rigaku Corporation, Japan) with $\mathrm{Cu}-\mathrm{K} \alpha$ radiation $(\lambda=1.542 \AA)$ at $40 \mathrm{kV}$ and $250 \mathrm{~mA}$. The XRD patterns were recorded over the $2 \theta$ range of $4-40^{\circ}$ at a speed of $2 \mathrm{deg} \mathrm{min}^{-1}$.

\section{Results and discussion}

\subsection{Mean size of ANPs}

Figure 1 shows effects of surfactant concentration on mean size and PDI of the precipitated ANPs. Compared with the ANPs without using surfactant, adding $0.5 \%$ surfactant (in proportion of amylose solution volume) led to the formation of smaller ANPs, but, with the increase in the amount of surfactant, the sizes of the ANPs increased. Similar trend was also observed by Hebeish et al [17] in maize SNPs. This phenomenon could be because most of amylose molecules were precipitated before interacting with surfactant when a small amount of surfactant was present in ethanol. The surfactant molecules absorbed at the surface of the formed nanoparticles

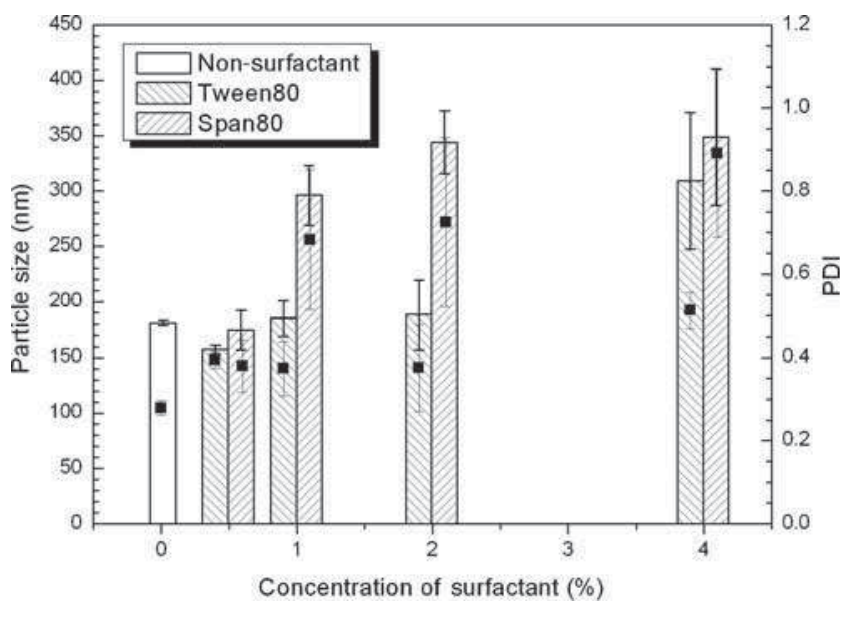

Figure 1. Effects of surfactant concentration on mean size (column) and PDI (solid square) of precipitated ANPs.

would provide steric hindrance to limit growth of the particles. However, when concentration of surfactant was high, some amylose molecules acted with surfactant first, and these amylose molecules could result in large aggregates during precipitation due to chain aggregation with large interstitial space.

Figure 1 also shows that with the same amount of surfactant, the size of the ANPs using Tween80 was smaller than that using Span80. Being more hydrophilic than Span80, Tween80 interacts more strongly with amylose molecules. Thus, ANPs with smaller sizes were precipitated [8]. It was noted that the PDI values of the ANPs obtained by using higher concentration of Span80 $(\geq 1 \%)$ were close to or higher than 0.7 , indicating that these samples were colloidal suspensions which have a very broad size distribution and may contain large particles or aggregates.

To optimize surfactant efficiency, Tween 80 and Span80 were mixed with different ratios. The effect of hydrophiliclipophilic balance (HLB) on the size of precipitated ANPs was investigated. The concept of HLB can be used to characterize the relative affinity of surfactant for aqueous and oil phases [18]. The HLB values of Tween80/Span80 mixtures were calculated using

$\mathrm{HLB}_{\text {mix }}=\mathrm{HLB}_{x} x \%+\mathrm{HLB}_{y} y \%$,

where $\mathrm{HLB}_{x}$ and $\mathrm{HLB}_{y}$ are low HLB surfactant and high HLB surfactant, respectively, $x \%$ and $y \%$ are their mass percentages, respectively. The mean sizes and PDIs of the ANPs using Span80/Tween80 mixtures with different HLB values are shown in table 1 . The results indicated that the size of precipitated ANPs was dependent on HLB of surfactant. The Span80/Tween80 mixture with HLB of 12.33 allowed a minimum particle size to be achieved. Although some previous works involved using surfactants to prepare SNPs via precipitation [8,17], the effect mechanism of surfactant is not clear. More data and further studies are needed to elucidate the influence of surfactant nature and concentration on nucleation of small aggregates of starch molecules and aggregation of these nuclei. 


\subsection{Morphology of ANPs}

Figure 2 shows morphologies of the precipitated ANPs using $0.5 \%$ (in proportion of amylose solution volume) different surfactants and the one without surfactant. The ANPs without using surfactant were spherical in shape (figure 2a), the ANPs using Tween80 (figure $2 b$ ) showed smaller size but the shape was irregular; while for the ANPs using Span80 (figure 2c), the shape was similar to that of the ANPs using Tween80, but some particles combined together and formed short rod-like and platelet-like aggregates. The ANPs using Tween $80 /$ Span 80 mixture with HLB of 12.33 (figure 2d) also showed spherical shape.

It was noted that the sizes of the ANPs shown in figure 2 were larger than that measured by DLS presented in figure 1 . This is because the samples for DLS measurements were prepared by dispersing the precipitated ANPs in water through

Table 1. Effects of surfactant HLB on mean size and PDI of ANPs.

\begin{tabular}{lccc}
\hline $\begin{array}{l}\text { Tween80/ } \\
\text { Span80 (w/w) }\end{array}$ & HLB & $\begin{array}{c}\text { Mean size } \\
(\mathrm{nm})\end{array}$ & PDI \\
\hline $0 / 100$ & 4.30 & $174.8 \pm 17.7$ & $0.380 \pm 0.063$ \\
$25 / 75$ & 6.98 & $183.1 \pm 3.7$ & $0.402 \pm 0.032$ \\
$50 / 50$ & 9.65 & $173.1 \pm 2.2$ & $0.422 \pm 0.017$ \\
$75 / 25$ & 12.33 & $155.2 \pm 2.8$ & $0.340 \pm 0.032$ \\
$100 / 0$ & 15.0 & $157.4 \pm 3.8$ & $0.394 \pm 0.020$ \\
\hline
\end{tabular}

ultrasonication. Since the ultrasonic dispersing could dissociate aggregated nanoparticles and the precipitated ANPs were partially soluble in water, therefore, the sizes of ANPs measured by DLS were smaller. Scanning electron microscope (SEM) micrographs of the ANPs re-dispered in water are shown in figure 3 . The sizes of the particles in figure 3 were consistent with that determined by DLS as presented in figure 1.

\subsection{XRD spectra of ANPs}

As presented in figure 4, XRD spectrum of amylose granules showed a scattering pattern with major diffraction peaks at $15.1^{\circ}, 17.1^{\circ}$ and $17.9^{\circ}$ (a strong double peak), and $23.0^{\circ}$ of $2 \theta$, indicating that the amylose granules have typical A-type crystalline structure in which amylose chains are intertwined in left-handed double helices and these double helices are packed in a parallel fashion $[9,19]$. While for the precipitated ANPs, the XRD patterns showed that there were diffraction peaks at $13.5^{\circ}$ and $19.8^{\circ}$, suggesting that the precipitated ANPs possessed the V-type crystalline structure which is single, left-handed helices with an internal cavity where guest molecules can reside [20]. It is generally believed that the V-type crystalline structure is a result of amylose being complexed with substances such as aliphatic fatty acids, surfactants, emulsifiers, $n$-alcohols, glycerol and DMSO [21]. Thus, the structural transition from the A-type in the amylose granules to the V-type in the ANPs is understandable.
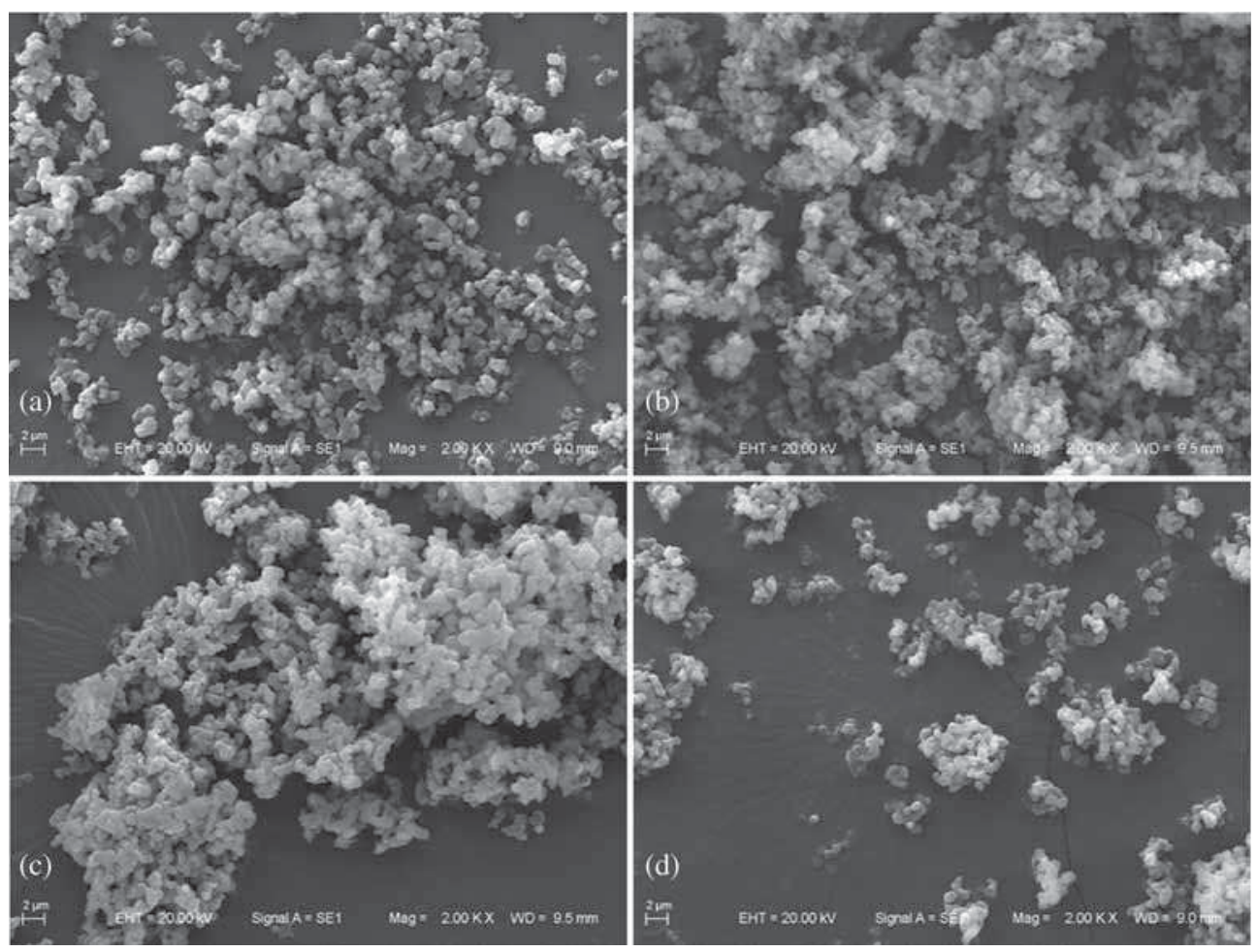

Figure 2. SEM micrographs of the precipitated ANPs: (a) without using surfactant; (b) using $0.5 \%$ Tween80; (c) using 0.5\% Span80; and (d) using 0.5\% Tween80/Span80 mixture with HLB of 12.33 . 

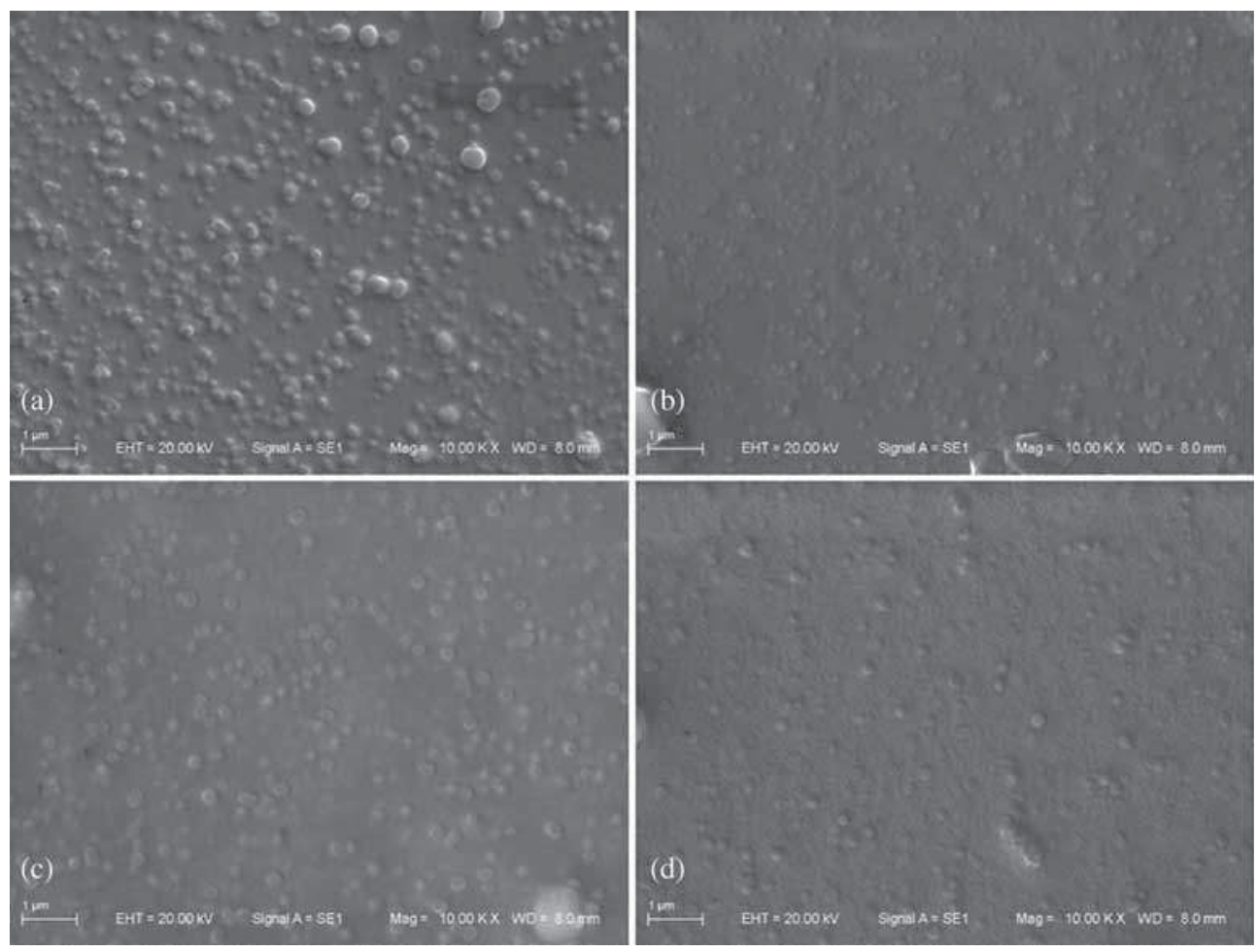

Figure 3. SEM micrographs of the ANPs re-dispersed in water: (a) without using surfactant; (b) using $0.5 \%$ Tween 80 ; (c) using $0.5 \%$ Span80; and (d) using $0.5 \%$ Tween $80 /$ Span 80 mixture with HLB of 12.33 .

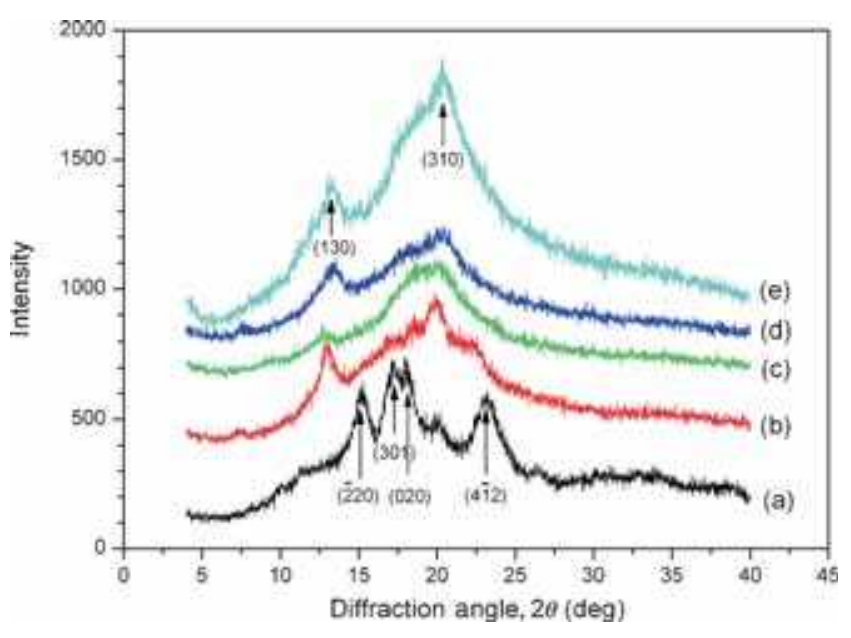

Figure 4. XRD spectra of (a) amylose granules; (b) the ANPs using Tween80; (c) the ANPs using Span80; (d) the ANPs using Tween80/Span80 mixture with HLB of 12.33; and (e) the ANPs without using surfactant.

The A-type crystalline structure was disrupted during amylose solution preparation (gelatinization), the dispersed amylose molecular chains re-coil into single helices with guest molecules in the internal cavity and formed the V-type crystalline structure during the precipitation. Since intensity of diffraction peak is related to crystallinity and the width at half height of a peak is inversely proportional to the crystallite size, the XRD patterns shown in figure 4 suggested that the crystallinity and crystallite size in the precipitated ANPs with various surfactants were different.

\section{Conclusions}

In this study, ANPs were prepared by precipitating amylose solution (using 90\% DMSO/10\% water mixture as solvent) with absolute ethanol containing Tween 80 and Span80 under controlled operating conditions. Surfactant type, concentration and hydrophilic-lipophilic balance had influence on the size of ANPs. The smallest ANPs with mean size of $155 \mathrm{~nm}$ determined by DLS were obtained by using $0.5 \%$ (in the proportion of the volume of amylose solution) of Tween80/Span80 mixture (HLB $=12.33$ ). There was no significant difference in morphologies among the precipitated ANPs. The precipitated ANPs possessed the V-type crystalline structure no matter whether the surfactants were used or not, but the crystallinity and crystallite size in the ANPs were dependent on the surfactants.

\section{Acknowledgement}

This work was supported by the National Natural Science Foundation of China (51273083). 


\section{References}

[1] Le Corre D, Bras J and Dufresne A 2010 Biomacromolecules 111139

[2] Le Corre D and Angellier-Coussy H 2014 React. Funct. Polym. 8597

[3] Kim H Y, Park S S and Lim S T 2015 Colloid Surf. B 126607

[4] Angellier H, Choisnard L, Molina-Boisseau S, Dole P and Dufresne A 2004 Biomacromolecules 51545

[5] Kim H Y, Park D J, Kim J Y and Lim S T 2013 Carbohydr. Polym. 98295

[6] Ma X F, Jian R J, Chang P R and Yu J G 2008 Biomacromolecules 93314

[7] Tan Y, Xu K, Li L L, Liu C, Song C L and Wang P X 2009 ACS Appl. Mater. Interfaces 1956

[8] Chin S F, Pang S C and Tay S H 2011 Carbohydr. Polym. 86 1817

[9] Kim J Y, Yoon Y W and Lim S T 2009 Carbohydr. Polym. 78 626

[10] Haaj S B, Magnin A, Petrier C and Boufi S 2013 Carbohydr. Polym. 921625
[11] Zhang Z, Shan H L, Sun J R, Weng Y, Wang X, Xiong J and Chen X S 2013 RSC Adv. 313406

[12] Sun Q J, Li G H, Dai L, Ji N and Xiong L 2014 Food Chem. 162223

[13] Schubert S, Delaney J T and Schubert U S 2011 Soft Matter 7 1581

[14] Dong Y, Chang Y J, Wang Q, Tong J and Zhou J 2015 Starch/Stärke 67365

[15] Cooremana F L, van Rensburg H and Delcoura J A $1995 \mathrm{~J}$. Cereal Sci. 22251

[16] Chamberlain E K and Rao M A 1999 Carbohydr. Polym. 40 251

[17] Hebeish A, Ei-Rafie M H, El-Sheikh M and Ei-Naggar M E 2014 J. Inorg. Organomet. Polym. 24515

[18] Manchun S, Dass C R and Sriamornsak P 2014 Carbohydr. Polym. 101650

[19] Pérez S and Bertoft E 2010 Starch/Stärke 62389

[20] Putseys J A, Lamberts L and Delcour J A 2010 J. Cereal Sci. 51238

[21] Famá L, Goyanes S and Gerschenson L 2007 Carbohydr. Polym. 70265 